\title{
A continuous growth model for plant tissue
}

\author{
Behruz Bozorg ${ }^{1}$, Pawel Krupinski ${ }^{1}$ and Henrik Jönsson ${ }^{1,2,3}$ \\ ${ }^{1}$ Computational Biology \& Biological Physics, Lund University, Sölvegatan 14A, SE \\ 22362 Lund, Sweden, ${ }^{2}$ Sainsbury Laboratory, University of Cambridge, Bateman \\ Street, CB2 1LF Cambridge, United Kingdom ${ }^{3}$ Department of Applied Mathematics \\ and Theoretical Physics (DAMTP), University of Cambridge, Cambridge, United \\ Kingdom \\ E-mail: henrik@thep.lu.se
}

\begin{abstract}
Morphogenesis in plants and animals involves large irreversible deformations. In plants, the response of the cell wall material to internal and external forces is determined by its mechanical properties. An appropriate model for plant tissue growth must include key features such as anisotropic and heterogeneous elasticity and cell dependent evaluation of mechanical variables such as turgor pressure, stress and strain. In addition, a growth model needs to cope with cell divisions as a necessary part of the growth process. Here we develop such a growth model, which is capable of employing not only mechanical signals but also morphogen dependent signals for regulating growth. The model is based on a continuous equation for updating the resting configuration of the tissue. Simultaneously, material properties can be updated at a different time scale. We test the stability of our model by measuring convergence of growth results for a tissue under the same mechanical and material conditions but with different spatial discretization. The model is able to maintain a strain field in the tissue during re-meshing, which is of particular importance for modelling cell division. We confirm the accuracy of our estimations in two and three dimensional simulations, and show that residual stresses are less prominent if strain or stress is included as input signal to growth. The approach results in a model implementation that can be used to compare different growth hypotheses, while keeping residual stresses and other mechanical variables updated and available for feeding back to the growth and material properties.
\end{abstract}




\section{Introduction}

Most of the higher plants acquire their shape as a result of growth since cell migration and apoptosis are not contributing to morphogenesis. The plant cells are surrounded by structually strong and tightly connected cell walls and thus the changes in cell neighborhood topology are mainly due to cell proliferation. High internal pressure in the cells is the driving force of growth while heterogeneous and anisotropic mechanical properties of the cell walls are instrumental in shape formation [3, 11]. A classic growth model, introduced by James A. Lockhart in 1965, suggests that cell extension is appearing when cells walls are under tension, generated by cell pressure above a threshold value [22]. Long term growth is, next to elastic and plastic deformation, a fundamental process of plant tissue morphogenesis leading to organ formation. On a microscopic level it is a complicated and carefully balanced process of mass deposition, stress relaxation and geometrical expansion [9]. The plant cell walls are main mediators of this process, where their composite material plays an important role. The primary cell walls consist of strong load bearing cellulose fibers connected by xyloglucan molecules and residing in a matrix of pectin molecules [27]. The plant cells can guide the synthesis of the cellulose fibers in directions following cortical microtubules (MTs) and thereby they can regulate the anisotropy of their wall material [41]. The microscopic description of plant cell wall growth is still a subject of active research [9], but a simplistic illustration of the process is given by the breakage of the xyloglucan molecules, leading to slippage between the cellulose fibers and then addition of new material in between [11]. More recent work has introduced the idea of non-homogeneous growth at hotspots [45]. Furthermore, changes in the pectin gel matrix, assumed to provide an isotropic mechanical contribution to wall mechanics, can contribute to changes in growth. For example, pectin de-methylesterification has been indicated in loosening cell wall elasticity and changing prior to growth initiation in the shoot meristem $[28,10]$. More recently asymmetric changes of pectins in different walls of the epidermal cells have been proposed to contribute to anisotropic growth in hypocotyls [29], but the regulation of growth following pectin modification may be quite complex [44]. In addition to the physical description of growth, also molecular signals (often termed morphogens [43]) are important for initiating, driving and arresting the growth process. An example is auxin, a growth hormone suggested to alter cell wall properties by different mechanisms to induce growth $[31,26,15,38]$. Also involvement of other hormonal and genetic factors in the growth process has been confirmed by altering the cell wall composition $[12,23]$. It is not yet fully understood which quantity is most important for generating growth response in different plant tissues. The explicitly directional mechanical quantities, such as stress and strain are thought of contributing mainly to specification of growth direction. The growth rate is usually attributed to the action of morphogens, but from a mechanical point of view stress or strain magnitude as well as strain energy could be equally well considered as cues for growth rate and it is possible that different combinations mechanical and molecular signals participate in different growth processes. 
Plant growth has been modeled at a variety of resolutions, from discrete and continuum descriptions at organ level down to microscopic scales of single cell walls. At the tissue and whole plant scale models are mainly descriptive in nature, where for example L-systems has been used to build models of plant structures [30, 7]. Growth models are often phenomenological in connecting growth to variables such as water uptake, nutrients or environmental factors [39]. Continuous tissue models where morphogen-driven growth has been combined with mechanics have been used to describe growth of leaves and flowers $[21,37,18]$. The mechanical contribution in these models, however, has often been limited to maintenance of the integrity of the tissue, while residual stresses have been disregarded by removal at each update step [19]. At the microscopic scale, interactions between molecular components have been hypothesized, resulting in predictions of larger scale mechanical behavior [13, 25], but experimental verifications at this resolution is still lacking. More recently, models at the cellular level, including molecular signals and continuum descriptions of mechanical properties of the cell walls, have been developed $[17,14,4,36]$. Tensional stress has been suggested as an input to guide the direction of cortical microtubules, and finite element models were essential to connect the predicted stress directions to the measured MT directions $[16,35]$. The stress feedback model was further shown to lead to robust initiation of anisotropic shape via anisotropic material properties [8], while the need for isotropic wall mechanics at the initiation of new organs was supported with another model [36]. The use of atomic force microscopy for measuring mechanical properties of cell walls in vivo has been useful for confirmation of predictions of such models [35, 36]. Also, a 3D finite element model was used to show that the molecular input to growth is transformed to neighboring cells by variable mechanical response of differently sized cells [4], and 3D mechanical stress models have been used to feed back to subcellular molecular behavior within cells [17].

With few exceptions [6, 4], the models at the cellular level have been dealing with small elastic deformations. Thus a reliable mathematical description of growth in terms of continuous mechanics and practical implementation of such description are crucial for relating these mechanical quantities to growth. Here we present a model of finite growth and its regulation by different signals in a plant tissue. The model is based on separation of growth and elastic deformation processes by introduction of a growth dependent zero stress configuration as time dependent reference point for the constitutive equations [42]. Our growth model can be used with any material model and discretization. Here, due to the almost planar shape of plant cell walls, we use the already developed material model for anisotropic planar elements based on Saint Venant-Kirchhoff strain energy $[16,35,8]$. We test different mechanical signals, e.g. strain or stress, which can be evaluated directly from the mechanical variables, as well as mechanically independent signals, e.g. morphogens, as inputs to our growth model. When mechanical signals are used their principal directions and values are hypothesized to regulate both the direction and rate of the growth. 


\section{Methods}

Plant tissue, as a mechanical system, is restricted by relatively stiff walls with a particularly rapid elastic response to any changes in forces and material properties. This is necessary for the plants since they have to keep the cell walls intact under high stresses generated by the turgor pressure driving the growth [11,3]. On the other hand, due to the relatively long time it takes for molecular processes to produce macroscopic changes, growth is considerably slower than the elastic response. We will take advantage of this difference in time scales and assume that at each time step of the growth the stress field is statically balanced. In this section we first discuss the fundamental force balance laws and derive the quasi-static equation of motion. Next we introduce the continuous growth equation and test its stability by comparing its performance over different spatial discretization. Then we present equations explicitly including parameters by which different signals can regulate principal directions and rates of the growth. Special care is taken for describing cell division and the related re-meshing process. Although our model does not depend on a specific strain energy description, we derive the strain energy for an anisotropic material that is used in our simulations based on a discretization of the tissue into triangular planar elements.

\subsection{Balance laws and the quasi-static equation of motion}

Deriving the balance laws of mass and energy is challenging since these are not conserved quantities during growth. Energy is constantly provided to a living tissue, and mass is added into the growing tissue continuously. However, given that primary plant cell walls often grow in a plane with thickness of the walls staying constant we assume that the density, $\rho$, of the tissue is constant during growth described by the material derivative

$$
\frac{d \rho}{d t}=0
$$

The balance of angular momentum is satisfied by stress tensor symmetry

$$
T_{i j}=T_{j i}
$$

and the balance of linear momentum gives Cauchy's first law of motion

$$
\rho \frac{d \mathbf{V}}{d t}+\eta \mathbf{V}-\nabla \cdot \hat{\mathbf{T}}-\rho \mathbf{b}=0
$$

where $\mathbf{V}$ is the velocity, $\hat{\mathbf{T}}$ is the Cauchy stress tensor and $\mathbf{b}$ represents the body forces. The term $\nabla . \hat{\mathbf{T}}$ gives traction generated by stress divergence and $\eta \mathbf{V}$ is the damping force resulting from viscosity of the medium. Deposition of new material to a plant cell wall is much slower than the elastic response of the material that moves the material points toward their equilibrium position. This difference is large enough to let us assume that the material points are always in their mechanical equilibrium in which the forces are balanced

$$
\nabla \hat{\mathbf{T}}+\rho \mathbf{b}=0 .
$$


Since we are interested in finding the equilibrium configuration and not the dynamics of it we use over-damped, Langevin dynamics in which the acceleration term is negligible and the velocity field of the material points is determined by the yet unbalanced traction and body forces

$$
\eta \mathbf{V}=\nabla \cdot \hat{\mathbf{T}}+\rho \mathbf{b}
$$

or

$$
\frac{d \mathbf{X}}{d \tau}=\nabla \cdot \hat{\mathbf{T}}+\rho \mathbf{b}
$$

where $\tau=t / \eta$ is time during which the material configuration $\mathbf{X}$ moves towards its equilibrium $\mathbf{X}_{e q}$. The viscosity of the media $\eta$ is for our purpose the parameter controlling the rate of convergence and does not have a physical meaning. Our final result does not depend on this parameter as we do not take the dynamics into account in the quasi-static case. The equilibrium can be described by the integral

$$
\mathbf{X}_{e q}=\mathbf{X}(\tau=0)+\left.\int_{0}^{\infty}[\nabla \cdot \hat{\mathbf{T}}+\rho \mathbf{b}] d \tau\right|_{\mathbf{X}_{0}},
$$

where $\mathbf{X}_{0}$ is the material resting configuration and is stated here to emphasize the dependency of the integral upon this configuration. Ideally, the integral in Eq. 7 should be evaluated until Eq. 4 is satisfied (here and in similar expressions below). Note that $\tau$ is an artificial time and the infinite limit of the integral is used to allow the integrand to become zero independent on a specific stopping criterium. $\mathbf{X}(\tau=0)$ is the configuration from which we start the integration and is equivalent to neither $\mathbf{X}_{0}$ nor $\mathbf{X}_{e q}$. Generally the uniqueness of an equilibrium configuration is not guaranteed and specifically for complicated configurations it is likely to have several equilibria. However, if the distance between $\mathbf{X}(\tau=0)$ and $\mathbf{X}_{e q}$ in phase space is small enough, it is not likely to encounter more than a single equilibrium configuration during the integration. We can reduce the size of the growth-related time step such that this uniqueness is achieved throughout the integration interval, as will be described below. This interval connects two closely related equilibrium configurations in two consecutive time-points, where in each of the configurations Eq. 4 is satisfied. In practice, Eq. 7 can be used to set a numerical recipe for deriving an approximate equilibrium configuration. This leads to the iterative update equation

$$
\mathbf{X}_{n+1}=\mathbf{X}_{n}+\left[\nabla \cdot \hat{\mathbf{T}}\left(\mathbf{X}_{n}\right)+\rho \mathbf{b}\left(\mathbf{X}_{n}\right)\right] \delta \tau,
$$

where $X_{n}$ is the material configuration at iteration $n$. This update should be iterated until

$$
\left\|\nabla \cdot \hat{\mathbf{T}}\left(\mathbf{X}_{n}\right)+\rho \mathbf{b}\left(\mathbf{X}_{n}\right)\right\|<v_{c}
$$

where $\|\mathbf{x}\|$ is the maximal absolute value of the elements in $\mathbf{x}$ and $v_{c}$ is an equilibrium velocity threshold. The stopping criterion in Eq. 9 assures closeness of the final configuration to the equilibrium state, which we assume is unique within the neighborhood of integration. 


\subsection{The growth tensor}

The next step is to add growth, implemented as a deformation of the resting configuration $\mathbf{X}_{0}$. The mapping between the resting and current configurations, given as elastic deformation, is provided by the deformation gradient tensor, $\hat{\mathbf{F}}_{e}$, defined by

$$
\hat{\mathbf{F}}_{e}=\frac{\partial \mathbf{X}}{\partial \mathbf{X}_{0}}
$$

where $\mathbf{X}$ and $\mathbf{X}_{0}$ are the current and resting configurations, respectively (Fig. 1). The subscript $e$ is there to emphasis that this mapping describes only the elastic deformation. We define growth as a continuous update of the resting configuration that is assumed to be stress free but not necessarily compatible. A source of incompatibility is the non-uniform growth field under which adjacent elements can have different principal growth directions and rates. The overall mapping between initial resting configuration

and current configuration, $\hat{\mathbf{F}}_{e g}$, is given by the product of the growth and deformation gradient tensors

$$
\hat{\mathbf{F}}_{e g}=\hat{\mathbf{F}}_{e} \hat{\mathbf{F}}_{g}(t),
$$

where $\hat{\mathbf{F}}_{g}(t)$ is the finite growth tensor at time $t$. This multiplicative decomposition has been widely used when modeling tissue growth [2, 40, 33].

We introduce growth as an update of the resting configuration after an infinitesimal time step, $\delta t$, as

$$
\begin{aligned}
\mathbf{X}_{0}(t) \rightarrow \mathbf{X}_{0}(t+\delta t) & =\hat{\mathbf{F}}_{g}(t+\delta t) \mathbf{X}_{0}(0) \\
& =\mathbf{X}_{0}(t)+\delta \mathbf{X}_{0} \\
& =\left[\hat{\mathbf{I}}+\hat{\mathbf{f}}_{g}(\delta t)\right] \mathbf{X}_{0}(t),
\end{aligned}
$$

where $\mathbf{X}_{0}(0)$ is the initial resting configuration, which is constant, and $\hat{\mathbf{f}}_{g}$ is the differential growth tensor. The relation between $\hat{\mathbf{F}}_{g}$ and $\hat{\mathbf{f}}_{g}$ is given by

$$
\frac{\partial \hat{\mathbf{F}}_{g}}{\partial t}=\hat{\mathbf{f}}_{g} \hat{\mathbf{F}}_{g}
$$

which in integral form becomes

$$
\mathbf{F}_{g}(t)=\exp \left[\int_{0}^{t} \hat{\mathbf{f}}_{g}\left(t^{\prime}\right) d t^{\prime}\right] .
$$

The evolving resting shape and its growth dynamics is described by the difference equation

$$
\mathbf{X}_{0}(t+\delta t)-\mathbf{X}_{0}(t)=\hat{\mathbf{f}}_{g} \mathbf{X}_{0}(t) \delta t
$$

leading to the differential equation

$$
\frac{\partial \mathbf{X}_{0}}{\partial t}=\hat{\mathbf{f}}_{g} \mathbf{X}_{0}(t)
$$

with the solution

$$
\mathbf{X}_{0}(t)=\hat{\mathbf{F}}_{g}(t) \mathbf{X}_{0}(0)
$$


The differential growth tensor $\hat{\mathbf{f}}_{g}$ generally depends on time and growth signals. In these cases Eq. 16 becomes more complicated, but in the numerical update via Eq. 17 we need to only update $\hat{\mathbf{f}}_{g}$ at each time step.

The rest configuration $\mathbf{X}_{0}$ is piecewise compatible, i.e. each element is compatible within itself. Incompatibility exists at the junctions between elements. In the case of planar elements these junctions are the edges of elements whereas for three-dimensional elements, e.g. tetrahedrons, faces represent the junctions. In practice, elements are connected via common nodes at the corners. This by itself constrains all elements in the tissue to stay intact in the current configuration. However, each element in the resting configuration is allowed to be disconnected and stress free.

\subsection{Continuous growth}

The current equilibrium configuration, $\mathbf{X}_{e q}=\mathbf{X}_{e q}\left(\hat{\mathbf{F}}_{g}, \mathbf{M}\right)$, is a function of the growth tensor and a vector of all material parameters, $\mathbf{M}$, and its time derivative can be expressed as

$$
\frac{d \mathbf{X}_{e q}}{d t}=\frac{\partial \mathbf{X}_{e q}}{\partial \hat{\mathbf{F}}_{g}} \frac{\partial \hat{\mathbf{F}}_{g}}{\partial t}+\frac{\partial \mathbf{X}_{e q}}{\partial \mathbf{M}} \frac{\partial \mathbf{M}}{\partial t},
$$

where the subscript $e q$ is to stress that the derivative should be evaluated when all the material points are at equilibrium. Assuming that for a short time interval material properties do not change, the source of growth comes solely from evolution of the resting shape and we keep only the first term on the right hand side. By using Eq. 15 we have

$$
\frac{d \mathbf{X}_{e q}}{d t}=\frac{\partial \mathbf{X}_{e q}}{\partial \hat{\mathbf{F}}_{g}} \hat{\mathbf{f}}_{g} \hat{\mathbf{F}}_{g}
$$

This is the growth equation of a slow process compared to the elastic deformation in

which the material properties do not change. Changes in material properties can then be added in between small growth updates when time is discretized.

\subsection{Time discretization of growth}

In a numerical algorithm we must make sure that the current shape is at the mechanical equilibrium at each time step. For a small step in time, $\delta t$, from Eq. 21 we get

$$
\mathbf{X}_{e q}(t+\delta t)-\mathbf{X}_{e q}(t)=\frac{\partial \mathbf{X}_{e q}}{\partial \hat{\mathbf{F}}_{g}} \hat{\mathbf{f}}_{g} \hat{\mathbf{F}}_{g} \delta t
$$

or

$$
\mathbf{X}_{e q}(t+\delta t)=\mathbf{X}_{e q}\left(\hat{\mathbf{F}}_{g}\right)+\frac{\partial \mathbf{X}_{e q}}{\partial \hat{\mathbf{F}}_{g}} \delta \hat{\mathbf{F}}_{g}=\mathbf{X}_{e q}\left(\hat{\mathbf{F}}_{g}+\delta \hat{\mathbf{F}}_{g}\right),
$$

which from Eqs. 7 and 17 can be rewritten as

$$
\mathbf{X}_{e q}(t+\delta t)=\mathbf{X}_{e q}(t)+\left.\int_{0}^{\infty}[\nabla . \hat{\mathbf{T}}+\rho \mathbf{b}] d \tau\right|_{\left(\hat{\mathbf{I}}+\hat{\mathbf{f}}_{g} \delta t\right) \mathbf{X}_{0}(t)},
$$




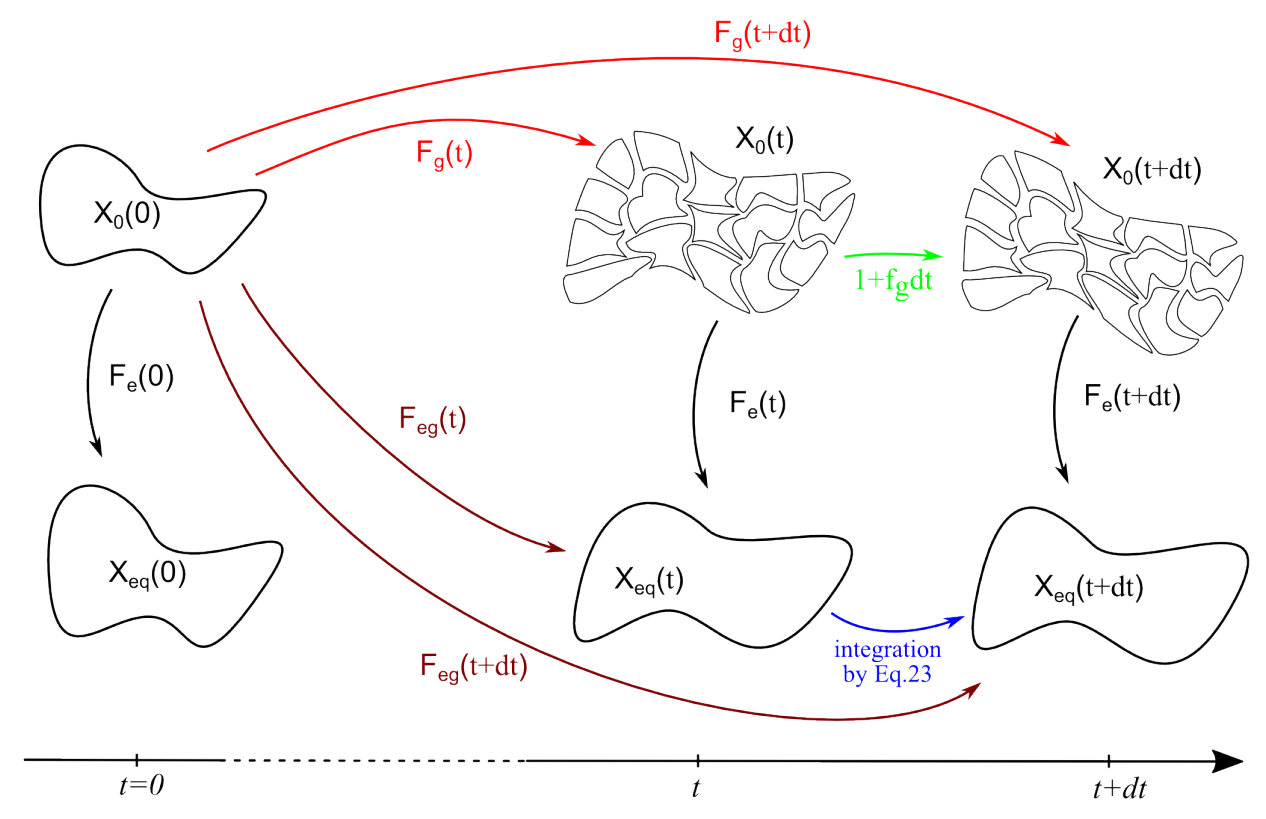

Figure 1. Illustration of the growth process. The resting configuration might be compatible at growth-time zero but can become incompatible later, while the current configuration stays compatible. The integration takes place between two consecutive growth times. All variable notation is defined in the main text.

or

$$
\mathbf{X}_{e q}^{n+1}=\mathbf{X}_{e q}^{n}+\left.\int_{0}^{\infty}[\nabla \cdot \hat{\mathbf{T}}+\rho \mathbf{b}] d \tau\right|_{\left(\hat{\mathbf{I}}+\hat{\mathbf{f}}_{g} \delta t\right) \mathbf{X}_{0}^{n}} .
$$

The blue arrow in Fig. 1 illustrates the update of the equilibrium configuration between two consecutive time points. The integral on the right hand side of Eq. 25 can be evaluated more easily if the consecutive equilibrium configurations are close to each other. This is equivalent to choosing a small step size for the growth. The growth update does not include changes in material properties (Eqs. 20-21), which can be added in between the growth updates at independent rates similar to what has been suggested previously (e.g. $[16,8])$.

\subsection{The growth signal}

A possibility for applying various inputs that connect the growth process with mechanical or biochemical signals is required given the complexity of the growth process. Assuming that the growth field generally can be inhomogeneous and anisotropic, the growth tensor can be defined as

$$
\hat{\mathbf{f}}_{g}=\sum_{i} \mathcal{F}\left(g_{i}\right) \mathbf{g}_{i} \mathbf{g}_{i}^{\mathcal{T}}
$$

where $g_{i}$ and $\mathbf{g}_{i}$ are the $i^{\text {th }}$ principal value and vector of growth signal in the resting configuration. $\mathcal{F}\left(g_{i}\right)$ is a function of the growth signal. This function can be non-linear and possibly very complicated due to the potentially complex growth processes involved. 
Here, as an example, we assume a simple piecewise linear relation between growth signal and rate given by

$$
\mathcal{F}\left(g_{i}\right)=k_{\text {rate }} \mathcal{R}\left(g_{i}-g_{t}\right)
$$

$g_{t}$ is the growth signal threshold above which growth occurs, $k_{\text {rate }}$ is the rate and $\mathcal{R}$ is the ramp function

$$
\begin{aligned}
& \mathcal{R}(x)=0 \quad \text { if } \quad x \leq 0 \\
& \mathcal{R}(x)=x \quad \text { if } \quad x>0 .
\end{aligned}
$$

Finally we formulate the general growth tensor as

$$
\begin{aligned}
\hat{\mathbf{f}}_{g} & =k_{\text {rate }} \sum_{i} \mathcal{R}\left(g_{i}-g_{t}\right) \mathbf{g}_{i} \mathbf{g}_{i}^{\mathcal{T}} \\
& =k_{\text {rate }}\left|\hat{\mathbf{F}}_{e}^{\mathcal{T}} \hat{\mathbf{F}}_{e}\right|^{-1} \sum_{i} \mathcal{R}\left(G_{i}-G_{t}\right) \hat{\mathbf{F}}_{e}^{\mathcal{T}} \mathbf{G}_{i} \mathbf{G}_{i}^{\mathcal{T}} \hat{\mathbf{F}}_{e} .
\end{aligned}
$$

where $G_{i}$ and $\mathbf{G}_{i}$ are the $i^{\text {th }}$ principal value and vector of growth signal and $G_{t}$ is the growth threshold, all given in the current configuration. $F_{e}$ is the elastic deformation gradient tensor. For stress or strain-based growth the growth tensor, can be defined as

$$
\mathbf{f}_{g}=k_{\text {rate }} \sum_{i} \mathcal{R}\left(S_{i}-S_{t}\right) \mathbf{S}_{i} \mathbf{S}_{i}^{\mathcal{T}}
$$

where $S_{i}$ and $\mathbf{S}_{\mathbf{i}}$ are the $i^{t h}$ principal value and vector of strain or stress in the resting configuration. $S_{t}$ is the strain or stress threshold for growth. This is more general than

the growth equation, $f_{g}=k_{\text {rate }} \hat{\mathcal{R}}\left(\hat{\mathbf{S}}-\hat{\mathbf{S}}_{\mathbf{t}}\right)$ used in other models [6], where $\hat{\mathbf{S}}$ and $\hat{\mathbf{S}}_{\mathbf{t}}$ are tensors of strain or stress and corresponding threshold, respectively, and $\hat{\mathcal{R}}$ is a tensor ramp function. In particular, Eq. 26 is invariant under rotation and remains unchanged in different coordinate systems.

In our model the growth signal can be stress, strain or any mechanics-independent factor, e.g. a morphogen concentration or gradient. It is also possible to have a growth scenario in which a combination of different factors regulates the growth. This can allow to extend previously proposed phyllotaxis models where the morphogenesis pattern is spontaneously forming by a combination of hormonal and mechanical signals $[24,17]$.

\subsection{Growing the elements in practice}

Application of Eq. 18 on the resting configuration for each of its elements after using Eq. 29 leads to a growth equation for each element edge. The growth rate of each element edge should be proportional to its resting length and a monotonic function of the principal strain values above the growth threshold, i.e. given by

$$
\begin{aligned}
\frac{\partial \mathbf{L}}{\partial t} & =\hat{\mathbf{f}}_{g} \mathbf{L} \\
& =k_{\text {rate }} \sum_{i} \mathcal{R}\left(g_{i}-g_{t}\right) \mathbf{g}_{i} \mathbf{g}_{i}^{\mathcal{T}} \mathbf{L} .
\end{aligned}
$$


For the $i^{\text {th }}$ component of the edge element

$$
\frac{\partial L_{i}}{\partial t}=k_{\text {rate }} \mathcal{R}\left(g_{i}-g_{t}\right) L_{i}
$$

Here we have assumed that the growth rates of the components of the element edges are proportional to their size and strength of the growth signal in the corresponding direction. Note that in this description we have used a simple factor for the growth, $k_{\text {rate }}$ and a vector description of growth signal in the ramp function, $\mathcal{R}\left(g_{i}-g_{t}\right)$. This allows for complex description of the growth process, which could be extended further with replacing $k_{\text {rate }}$ with complex functions of molecular and mechanical variables. At the same time this does not prevent the model from following simpler growth descriptions such as a scalar material yield description without a directional component. For example, a growth hormone like auxin can be implemented by replacing $k_{\text {rate }}$ with a function of its concentration, or by using the (scalar) concentration value as growth signal $\left(g_{i}\right.$, the same in all directions), or replacing the threshold $g_{t}$ with a function of the concentration. Similarly, the influence of cellulose fibers may be included by changing growth rules differently in different directions depending on the fiber orientations.

\subsection{Residual stresses}

In the case of an incompatible growth process we expect arise of the residual stresses in the tissue, i.e. stresses that are remaining in the tissue after loading forces are removed. Then the stress, $T$, in Eqs. 3-9 includes both residual, $\hat{\mathbf{T}}_{r}$, and loading, $\hat{\mathbf{T}}_{l}$, stresses

$$
\hat{\mathbf{T}}=\hat{\mathbf{T}}_{l}+\hat{\mathbf{T}}_{r} .
$$

Note that residual stresses are divergence free. This follows from the assumption that there are no external forces in the reference configuration [32]. Eqs. 3-9 are also true for net stresses, i.e.

$$
\nabla \cdot \hat{\mathbf{T}}_{l}+\rho \mathbf{b}=0
$$

and

$$
\nabla \cdot \hat{\mathbf{T}}_{r}=0 .
$$

The superposition principle allows us to add up the above equations and Eq. 35 provides a recipe for evaluating the residual stresses by

$$
\mathbf{X}_{e q}=\int_{0}^{\infty}[\nabla . \hat{\mathbf{T}}] d \tau \mid \mathbf{x}_{0}\left(T_{t o t}\right)
$$

where $T_{t o t}$ is the total growth time and

$$
\mathbf{X}_{0}\left(T_{t o t}\right)=\hat{\mathbf{F}}_{g}\left(T_{t o t}\right) \mathbf{X}_{0}=\left[\int_{0}^{T_{t o t}} \exp \left(\hat{\mathbf{f}}_{g} t\right) d t\right] \mathbf{X}_{0}
$$

Note that the tissue always experiences the overall stress field. Decomposing the stress into loading and residual components needs global information, which is not available to the cells. However, this decomposition can be of great importance when a stress field needs to be evaluated from the shape of the current configuration. When residual 
stresses exist, the geometrical information is not enough for the evaluation of the overall stress field. Eq. 37 can be used after each stage in the growth process to evaluate residual stresses. When this is done iteratively, the appearance and evolution of the residual stress field can be investigated.

\subsection{Cell division}

Growth often involves large deformations in which cells divide multiple times. Although a cell division includes various processes, e.g. mitosis and microtubular bundle formation, in a mechanical cell wall model the division can be simplified to adding a new wall connected to existing walls. For the existing walls this leads to addition of new degrees of freedom to the tissue without changing the material properties or mechanical variables such as stress and strain fields (Fig. 2). At each cell division, new domains connected to the new daughter cells replace a domain of the tissue that belongs to the cell wall to be subdivided. The positioning of a new wall by the cell follows specific rules $[34,5]$. Generally, the new wall created at division connects to the old walls at points that do not respect the arrangement of the elements in the existing mesh. Assuming each cell wall is meshed with some elements (Fig. 2), the new

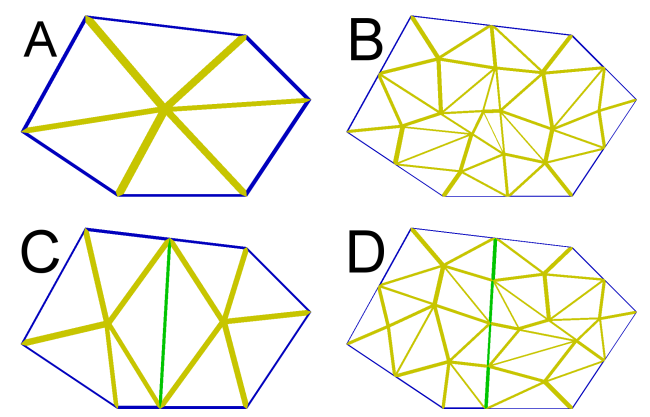

Figure 2. Cell division and re-meshing. Main walls are visualized using blue edges. A new main wall is created at division is displayed as a green edge. All the edges colored in yellow are the internal edges, which are the interfaces between triangular elements and their resting lengths must be estimated after re-meshing.

wall can divide the connecting cell walls regardless of their current meshes. This will require re-meshing after each cell division such that the new elements appear solely in walls connected to one of the daughter cells. Consequently the dimensions, strains and stresses for the new elements must be recalculated, and here we provide a recipe for minimizing the difference in strains before and after the division in individual cell walls.

2.8.1. Strain maintenance A division could be applied to the resting configuration of the cell wall if the elements are compatible, but this is not true after an incompatible growth process. Still it is possible to divide the mother cell wall and re-mesh the daughter cell walls in the compatible current configuration. As the resting configuration is essential for evaluating the strain energy, this needs to be estimated for each element 
based on the average strain field of the daughter cell walls, which has to be inherited from the mother cell wall. In such way the strain field in a wall does not go through a discontinuous change when a cell divides.

If the average Almansi strain tensor of the cell wall is known, it is possible to estimate the resting edges of elements from their edge vectors and eigenvalues and eigenvectors of the strain tensor. The Almansi strain in one dimension, $\epsilon_{E}$, is defined by

$$
\epsilon_{E}=\frac{1}{2}\left(\frac{l^{2}-L^{2}}{l^{2}}\right),
$$

where $l$ and $L$ are the current and resting lengths, respectively. We can invert this relation to calculate the resting length in terms of strain and current length

$$
L=\left[\left(1-2 \epsilon_{E}\right) l^{2}\right]^{\frac{1}{2}} \text {. }
$$

Similarly the Eulerian-Almansi finite strain tensor, $\hat{\mathbf{s}}$, is defined by

$$
\hat{\mathbf{s}}=\frac{1}{2}\left(\hat{\mathbf{I}}-\hat{\mathbf{F}}^{-\mathcal{T}} \hat{\mathbf{F}}^{-1}\right)
$$

where $F^{-1}$ is the inverse and $F^{-\mathcal{T}}$ is the inverse of the transpose of $F$. Inspired by Eq. 39, the resting length of each element edge is estimated by

$$
L=\left[\sum_{i}\left(1-2 s_{i}\right)\left|\mathbf{L}^{\mathcal{T}} \mathbf{s}_{i}\right|^{2}\right]^{\frac{1}{2}},
$$

where $s_{i}$ and $\mathbf{s}_{i}$ are the $i^{\text {th }}$ eigenvalue and eigenvector of the average Almansi tensor of the mother cell wall and $\mathbf{L}$ is the corresponding element edge vector in the current configuration.

Eq. 41 is equivalent to a transformation under the inverse of the average stretch component of the deformation gradient tensors of all elements of a cell wall.

\subsection{Saint Venant-Kirchhoff strain energy for anisotropic material and planar triangular elements}

The implementation of the models use strain energy based on the Saint Venant-Kirchhoff description

$$
W_{i s o}==\frac{\lambda}{2}(\operatorname{tr} \hat{\mathbf{E}})^{2}+\mu t r \hat{\mathbf{E}}^{2}
$$

where $\hat{\mathbf{E}}$ is the Green-Lagrange strain tensor and $\lambda$ and $\mu$ are the Lame coefficients of the material [8]. We assume plane stress condition where the Lame constants, $\lambda$ and $\mu$, can be expressed as

$$
\lambda=\frac{Y \nu}{1-\nu^{2}}, \quad \mu=\frac{Y}{2(1+\nu)} .
$$

Here $Y$ and $\nu$ are the Young's modulus and Poisson ratio that represent stiffness and incompressibility of the material, respectively. The modified version of Saint VenantKirchhoff model for anisotropic material is given by

$$
W=W_{\text {iso }}+W_{\text {aniso }},
$$


where

$$
W_{\text {aniso }}=\frac{\Delta \lambda}{2} \mathbf{a}^{\mathcal{T}} \hat{\mathbf{E}} \mathbf{a} \operatorname{tr} \hat{\mathbf{E}}+\Delta \mu \mathbf{a}^{\mathcal{T}} \hat{\mathbf{E}}^{2} \mathbf{a} .
$$

$\mathbf{a}$ is the anisotropy vector which shows the direction in the material with the largest elasticity constant. The $\Delta \lambda$ and $\Delta \mu$ are the differences between longitudinal and transverse Lame coefficients which are in turn related to the Poisson ratio and Young's modulus in longitudinal and transverse directions.

Elements are triangular plates under plane stress condition. It has been shown that such a description is appropriate for describing the main mechanical features of different plant tissues domains in an epidermal pressure model [8].

\section{Results}

To test the proposed growth model we perform simulations using a square patch of material, with triangular plate elements in the mesh and a Saint Venant-Kirchhoff strain energy model allowing for anisotropic material properties. Material parameters and stresses are chosen such that the elastic deformation is about 8 to $12 \%$. This value has been reported to be a relevant elastic deformation in plants [20].

\subsection{The growth model can be made highly independent on spatial discretization}

The stability of the growth model can be tested via comparing the resulting deformations for the same mechanical conditions but using different spatial discretization where the number of degrees of freedom of the tissue varies. First, we investigate the convergence properties of the area expansion on grown templates with different discretization resolution. A template with isotropic material and discretized at three different resolutions from coarse to fine is used (Fig. 3A). Forces are applied to grow the template to about double the size. In the test cases we applied isotropic loading forces (Fig. 3B), uniaxial loading forces (Fig. 3C), or forces generated by applying a pressure from one side (Fig. 3D).

Since growth is applied on the resting shape, we compared the area of the resting shape as a function of time of growth for different discretization resolutions (Figs. 3E-J). As described in the Methods section (Eq. 9), numerical simulations use an equilibrium threshold value $\left(v_{c}\right)$ to estimate when the tissues is close enough to an elastic equilibrium between each growth updates. Lowering $v_{c}$ leads to a convergence of the resting shape areas for the different meshes (cf. Figs. 3E-G with Figs. 3H-J where the value of $v_{c}$ has been lowered about one third in $\mathrm{H}$ and $\mathrm{I}$ and about twofold in $\mathrm{J}$ ). The convergence is particularly evident in the $2 \mathrm{D}$ simulations, where it is possible to reduce the growth error by decreasing $v_{c}$, at the cost of longer simulation times. For example, when comparing the difference between meshes with more than one order of magnitude difference in number of elements ( $N=31$ vs $N=518$ ), the area difference decreases from $20 \%$ and $15 \%$ (Figs. 3E and F) to $12 \%$ and $5 \%$ (Figs. $3 \mathrm{H}$ and $\mathrm{I}$ ) when the reduction in $v_{c}$ is less than one third. In these simulations the comparison is done after the area of the coarser 

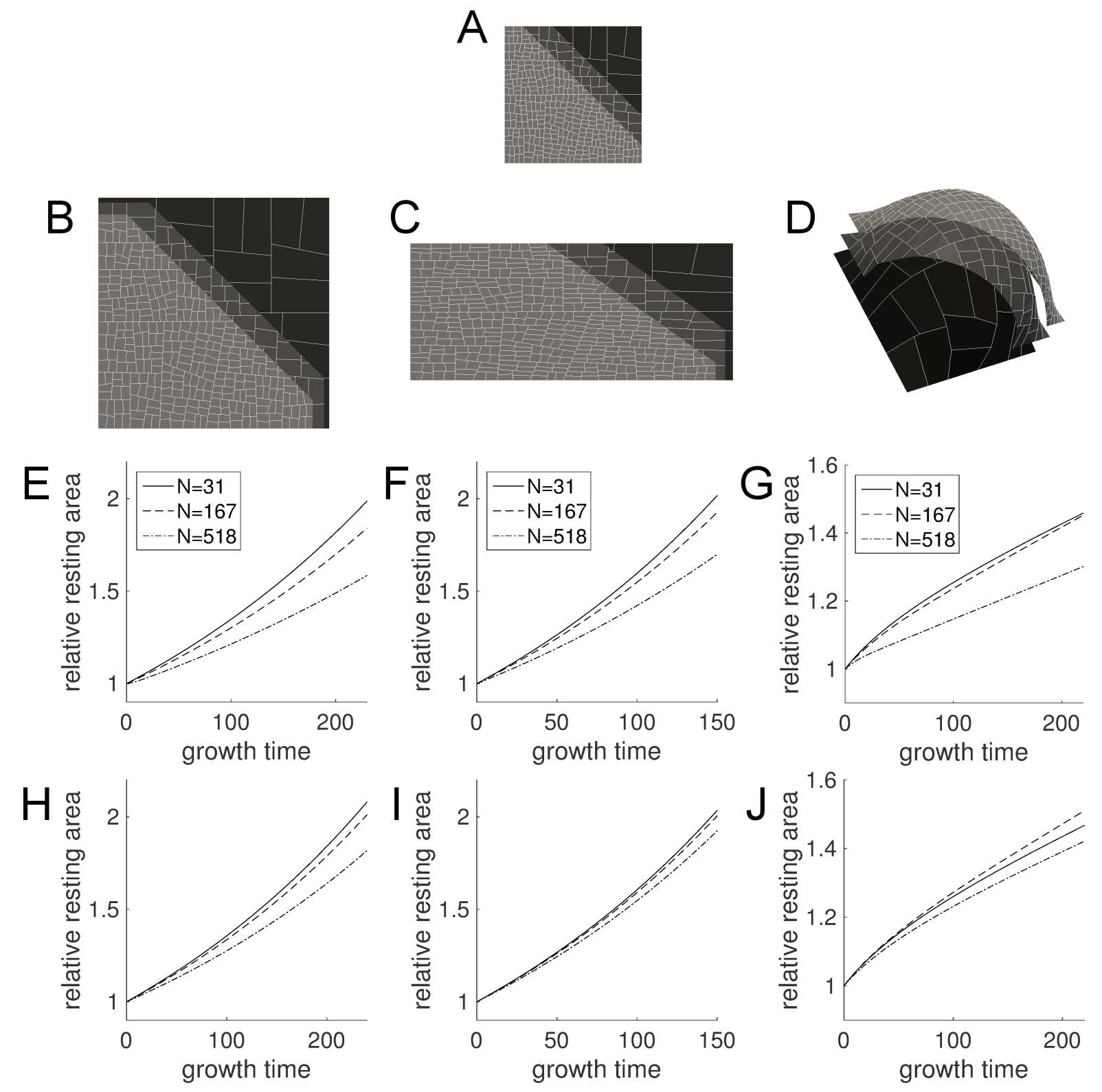

Figure 3. Growth and different spatial discretization. (A) A square template meshed in three different sizes (shown in different shades of gray) is used as initial configuration. (B, E, H) The template is grown isotropically (C, F, I) The template is grown uniaxially. (D, G, J) The template is grown in three dimensions by inflating it by applying pressure on one side. (B, C, D) The deformed templates after growth. (E-J) Relative resting areas versus growth time (different than simulation time) are presented for two different equilibrium thresholds, $v_{c}$. The equilibrium threshold value is 0.01 for $\mathrm{E}$ and $\mathrm{F}, 0.003$ for $\mathrm{H}$ and $\mathrm{I}, 0.001$ for $\mathrm{G}$ and 0.0004 for J. Elastic deformation $\approx 10 \%$ for $\mathrm{A}$ and $\mathrm{B}, \approx 0$ to $15 \%$ for $\mathrm{C}$.

mesh has been doubled. For the pressurized template (Figs. 3D, G and J) it becomes more complicated and the area convergence is not monotonic or might not happen at all. When applying pressure load to the template the shape and curvature is changing. Such shape changes are mesh dependent in our description using (planar) plate elements 
and a fine meshing will always be preferable if high resolution details of shape are to be investigated.

While it is possible to tune the difference in growth between different mesh resolutions, a finer mesh results in slower growth in general (using the same $v_{c}$ ). This is because after growing the elements, strain drops to lower values and it takes a while for the template to regain its quasi-equilibrium strain field. This time is longer when using a fine mesh. Consequently, principal growth values in Eq. 26 will be lower for a finer mesh when using the same equilibrium threshold.

In summary, we showed that continuous growth can be described using our framework, and that it is important to keep the system close to its elastic equilibrium for a mesh-independent result in describing the growth of the tissue.

\subsection{Different input signals for growth can lead to heterogeneous residual stresses}

Our approach allows for regulating growth by using mechanical or other signals (Eqs. 26 and 29). It is of interest to compare the situations where growth is controlled by either stress or strain signals since these can differ in the presence of material anisotropy in a tissue under anisotropic loading forces. Anisotropic forces are expected in plant

epidermal tissue and are a result from growth in neighboring tissue as well as the curvature of the tissue. The anisotropic alignment of cellulose fibers in key tissue domains in plants highlights the importance of such comparison, and although we will disregard dynamical material anisotropy in our simulations, feedback between stresses and material anisotropy can be of great importance for explaining robust material patterning in cells and tissues [8,35].

To investigate the difference between strain and stress-based growth we simulate an anisotropic patch of material loaded such that the stress is anisotropic (Figs. 4A and B). The material anisotropy and the stress anisotropy and their directions are chosen to give the maximal strain direction in the vertical direction and perpendicular to the horizontal maximal stress. Growing the tissue using Eq. 26 with either strain or stress as growth promoting signal results in different growth fields and completely different shapes (Fig. 4 $\mathrm{D}$ and $\mathrm{E}$ ). This provides an illustrative example of how altering the growth signal under the same mechanical conditions can produce different growth patterns and ultimately shapes. In particular it is important to discern between stress and strain as signals for growth [8].

In our approach it is possible to evaluate residual stresses if they exist (Eqs. 3337). After finite growth, the body forces can be removed and the tissue can be relaxed following Eq.37. The remaining stress field is a divergence free field [32]. The example simulations using stress and strain based growth with an anisotropic (but uniform) load lead to close to zero residual stresses (Figs. 4E and F). More interesting is to investigate residual stresses when a non-uniform growth signal is applied. We did this for a square patch using strain, stress or morphogen concentration as growth signal. The signal was applied non-uniformally (but isotropically), decreasing from left to right in the patch 

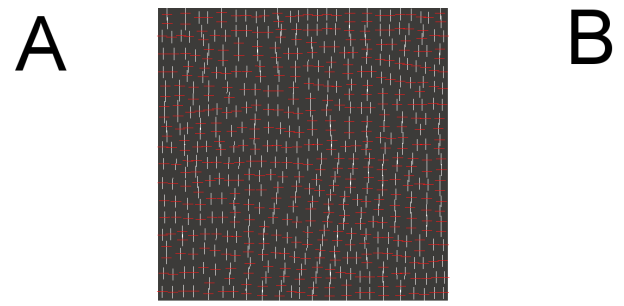
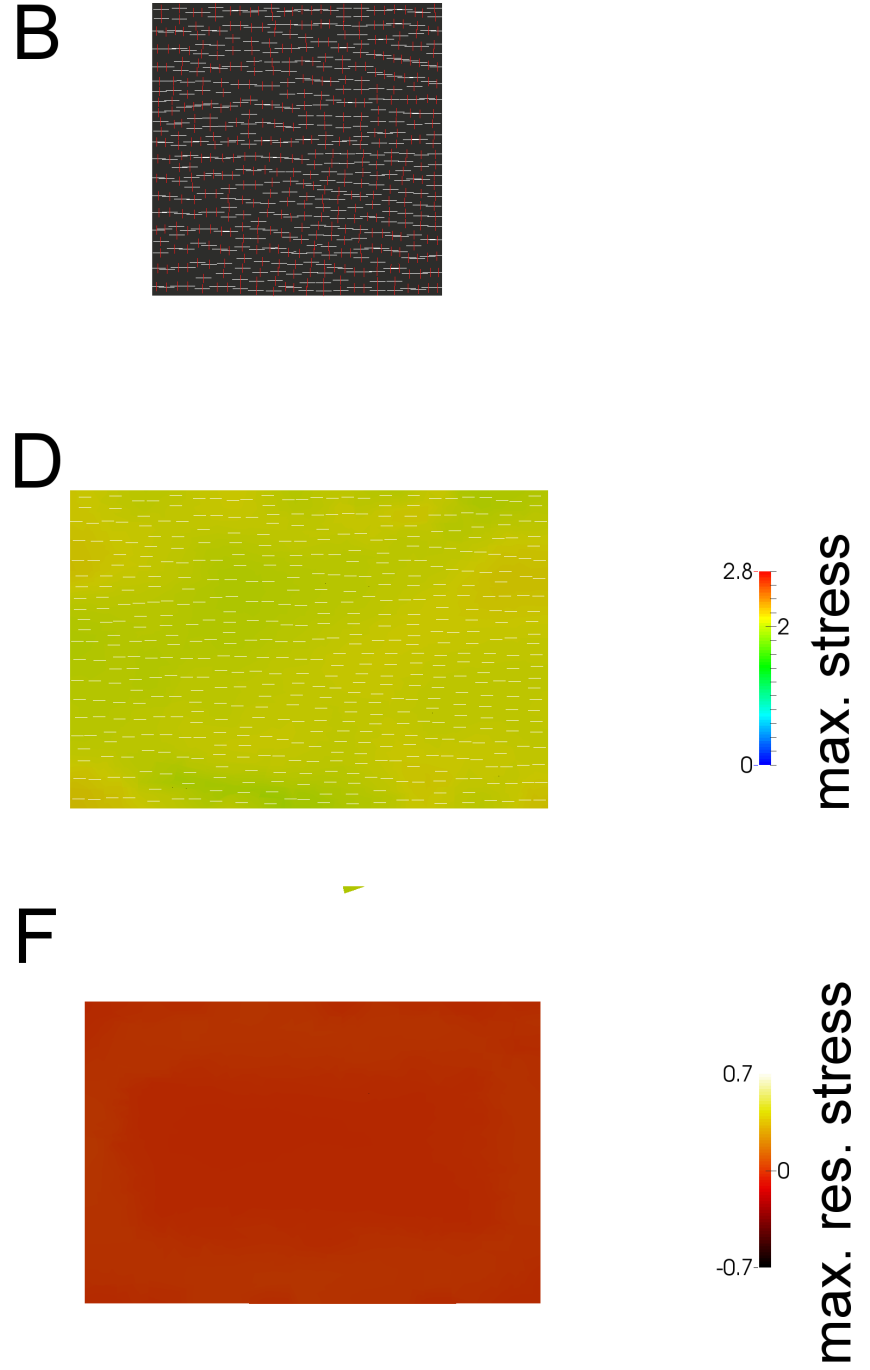

Figure 4. Shape changes, overall stress and residual stress fields resulting from different uniform growth rules. A, B) A patch of anisotropic material is loaded by anisotropic stress with a maximal load in the horizontal direction. The principal stress and strains are perpendicular since the material is anisotropic. The bars are showing the first (white) and second (red) eigenvectors of strain (stress) in A (B) and are scaled according to their corresponding eigenvalues. Growth is given by Eq. 29. C) Strain based growth results in vertical growth. D) Stress based growth generates horizontal growth. C, D) The color shows maximal principal value of the overall stress field resulting from body forces and residual stresses. E, F) The maximal principal value of the residual stress field, measured after removing the body forces and letting the tissues relax.

(Figs. 5A-C). The non-uniform signal results in a twisted shape of the patch after growth independent on the growth signal (Figs. 5D-F). The non-uniform signal results in both tensile and compressive residual stresses in different domains of the tissue that arise from keeping the tissue compatible and connected (Figs. 5G-I). However, when either strain or stress is used as growth signal, the residual stresses are clearly lower (Figs. 5G-H), indicating that the use of strain or stress signals for growth, as suggested e.g. in the 
simple Lockhart model, surpresses the accumulation of residual stresses.

A
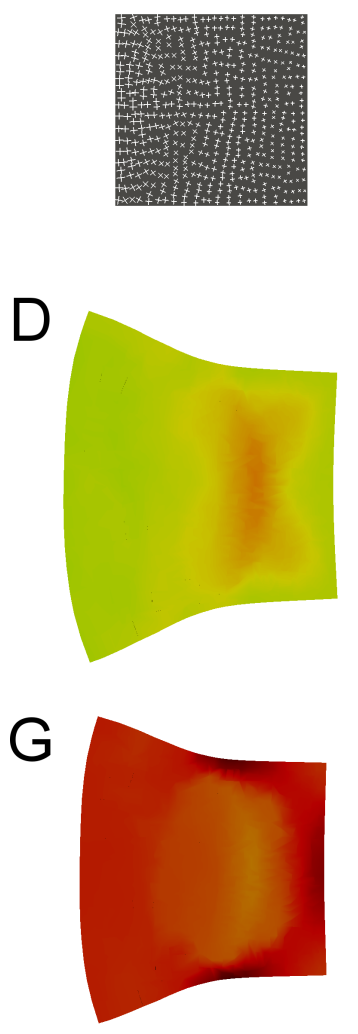

B
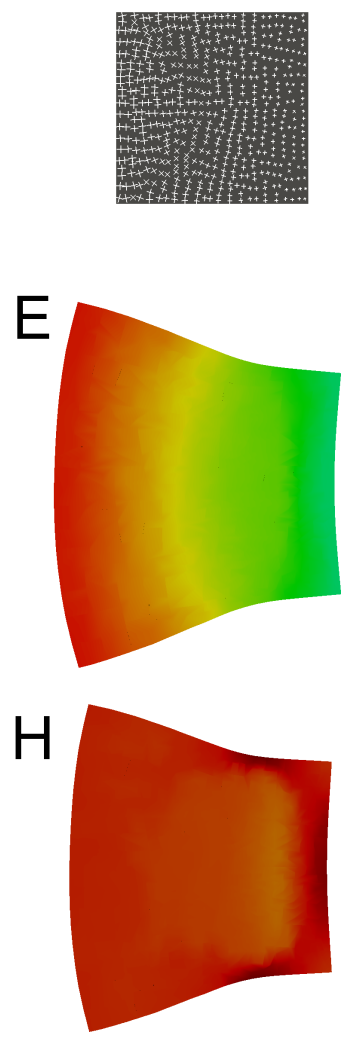
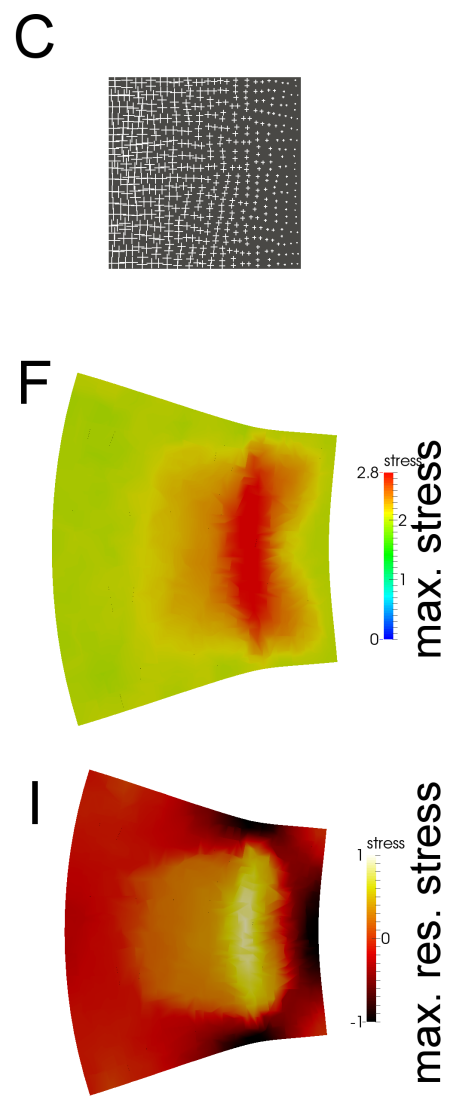

Figure 5. Shape changes, overall and residual stresses resulting from nonuniform growth fields generated by various growth signals. A) A square patch of non-uniform material with increasing young modulus is isotropically pressurized by an in-plane pressure resulting in a non-uniform strain field shown by white bars. Note that the strain field is isotropic and rotation of the crosses do not represent a preferable direction. B) The same patch as in (A) with a uniform material is pressurized isotropically but non-uniformly resulting in a non-uniform stress field shown by white bars. C) The same patch as in (A) and (B) of an isotropic material loaded by isotropic and uniform pressure. The growth signal is set due to a predefined pattern depicted by white bars resembling a morphogen concentration as a growth scalar. A-C) growth is implemented according to Eq. 29 using strain (A), stress (B) and morphogen signals (C). D-F) The loaded templates after growth using strain (D), stress (E) or morphogen concentration $(\mathrm{F})$. The color scale shows the pattern of principal overall stress. (the stress pattern in E is dominated by the applied non-uniform stress) G-I) The maximal principal value of the residual stress field, measured after removing the body forces and letting the tissues relax ( $\mathrm{G}$ - strain-based growth, H - stress-based growth, I morphogen-based growth).

We have shown the capabilities of the proposed model to employ different growth signals and demonstrated how this impacts the morphogenesis in some simple examples. We used this to explain how shapes can be generated by stress and strain signals and using non-mechanical inputs in the model. In particular, we evaluated how growth patterns relate to stress distributions, including how complex residual stresses can result 
when non-uniform growth is applied.

\subsection{The strain field is maintained during growth and cell division}

The difference between the resting and deformed configurations is given by the strain. Normally, by having the information about resting and current configurations the strain field can be calculated. However, as discussed in the Methods section, when cells divide new degrees of freedom need to be introduced, e.g. new nodes and edges for the resting configuration. This is done by interpolating the variables of the resting configuration in a finite element discretization. As we average the strain tensor over each cell wall, this tensor together with the values of the variables in the current configuration can be used to perform the necessary interpolation (Eq. 41).

For validating how well the method is able to predict a resting shape, we estimate the already known resting variables (here resting edges of triangular elements) from the average strain tensor of each cell wall and the current values of those variables. To do this we use elements of a collection of cells in a strained tissue and compare them with the corresponding exact values for a strained template in two and three dimensions (Fig. 6). For the 2D strain, the estimation is very accurate with an error (normalized mean square error) of about 0.055 , which is expected since the triangular elements building up the cell wall experience quite homogeneous forces in a single plane (Fig. 6C). In the 3D pressurized template, the error is slightly larger (0.058), as a result of some cell walls becoming curved. The errors in 3D are in general not high and the larger values come from curved cell surfaces, that are mainly located at the boundary where the cells are connecting the tissue to an infinitely stiff boundary in this simulation. Next, growth is added to the template using an isotropic material where the growth is regulated by a strain signal and where cells are allowed to divide according to a shortest path rule after reaching a threshold size [34]. The accuracy of cell division performance is evaluated by comparing the principal values of the average strain tensor of daughter cells with each other and those of their mother cell before and after cell division. Differences are very low (Fig. 7), and strikingly, even lower than in the non-growing situation (cf. Fig. 6). The reason is that when a cell divides there is an update of the estimate of the resting edge connected to a division plane, which decreases the error in further estimations. The error is generally small and smaller in the 2D simulation (Fig. 7D) than in the 3D simulation (Fig. 7E). The outliers in the 3D case are again coming from the divisions that happen adjacent to the stiff boundary where non-planar cell walls appear. We have shown that the error in estimating the resting state from the strain field and current configuration is quite low in our approach, including in the case of adding growth and cell division that leads to re-meshing of the wall discretization. Errors are increased close to the boundary, which can easily be mediated by adding additional tissue between the boundary and the region of interest or alternatively define more advanced boundary conditions. 

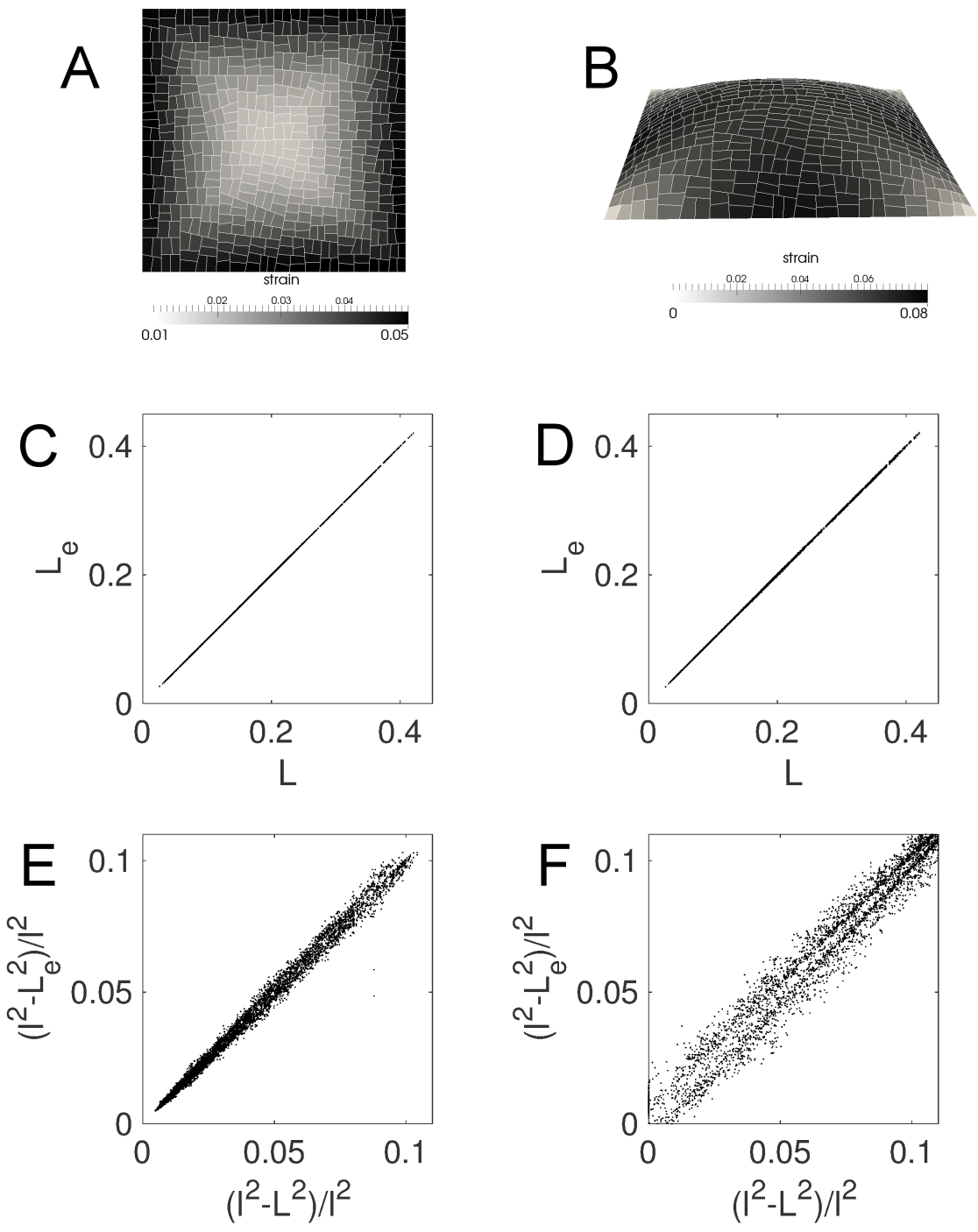

Figure 6. Resting shape estimation. A, C, E) A tissue strained by an in-plane isotropic stress. B, D, F) A tissue strained into 3D by a pressure force from one side. A, B) The maximal strain is visualized (gray-scale) on the current shape. C, D) The estimated, $L_{e}$ resting lengths of all edges are compared with the known resting lengths, $L$. E, F) The strain calculated from the estimated resting lengths vs the strain calculated from the known resting lengths.

\section{Discussion and Conclusions}

We have presented a way by which the processes of cell growth and division can be incorporated in finite element simulations of a biological tissue with particular focus on plant cell wall growth. The proposed formulation allows for simulating biomechanical events for a prolonged period of time and analyzing dynamic phenomena that extend over large periods of time. This includes all morphogenetic events that can now be approached with proper amount of mechanical details on cellular scale, and where feedback from gene regulatory, hormonal, mechanical and environmental cues can be 

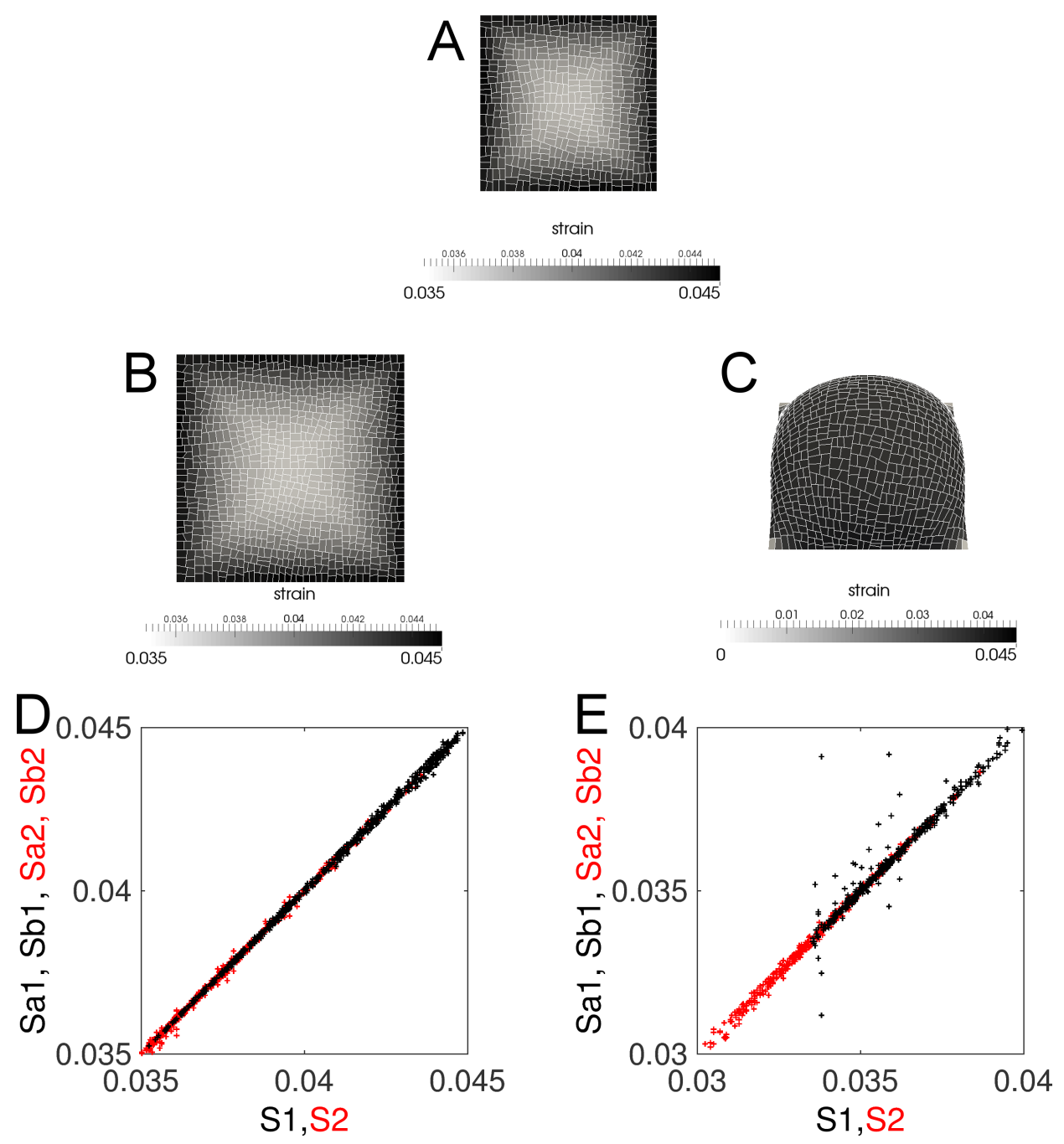

Figure 7. Growth and cell division. A) Initial tissue. B) The grown tissue with cell division in 2D by isotropic forces applied. C) The tissue grown using a pressure force from below. A-C) The magnitude of the principal strain is displayed (gray-scale). D) Normalized mean square error (NMSE) is $0.002 \mathrm{E}$ ) Error (NMSE) is 0.02 (including the outliers) D-E) S1 and S2 are the first and second principal values of strain in the mother cell before division, S1a and S2a are the principal values of strain in one of the daughter cells labeled with "a" after division and similarly S1b and S2b are the same values in the other daughter cell that is labeled with "b".

incorporated at levels of material properties as well as for growth rates and thresholds.

Inclusion of both growth and cell division causes several complications, which have to be carefully taken care of in order for a model to present consistent results. In particular, we show that our description of growth, when implemented using Saint Venant-Kirchhoff strain energy on triangular plates, provides a mesh-independent growth (Fig. 3). Importantly, this includes the remeshing appearing at cell division, where we show how to preserve the strain field in dynamical remeshing (Fig. 7). Further, we show how a variety of growth signals, including stress, strain or morphogens can be applied by altering mechanical properties of the cell walls, and how the residual stresses 
that result from different inputs can be identified. In particular we show how strain and stress based growth leads to less residual stresses, while a morphogen-based growth can lead to both tensional and compressing residual stresses even if the tissue is pressurized and only contain tensional stresses driving the growth (Fig. 4). We have used a simplistic linear growth rate, inspired by the Lockhart model of growth [22], but our description allows for nonlinear inputs to growth rates and other parameters leading to growth. The current formalism would for example allow for adding growth in a consistent way to nonlinear changes of material properties from molecular or stress inputs, as previously has been applied in non-growth simulations where there was a nonlinear feedback from the plant hormone auxin on Young's modulus [17], or a nonlinear feedback on material anisotropy from principal stress directions [16,8]. Also, the use of a specific strain energy formalism and triangular springs in our simulations is to exemplify possible applications, while the derivations presented in the Methods section are more generally applicable. While we have used examples of surfaces in two and three dimensions, there is nothing preventing the construction of tissues of $3 \mathrm{D}$ cells surrounded by $2 \mathrm{D}$ wall elements. Notably, we use two edges to connect two cell walls in our current description, and the 3D extension will need to adopt to connecting several cells by multiple edges and using two faces per mesh element to connect neighboring cells.

Our approach has similarities with some recent efforts of modeling plant cell wall growth $[16,34,19,1,4,6]$. In several of these examples, 2D tissues or surfaces in 3D were described $[16,34,1]$, where the walls were represented by $1 \mathrm{D}$ edge elements which cannot represent the material complexity described using our 2D descriptions of cell wall mechanics. An extension to this was to use proper $2 \mathrm{D}$ descriptions of the cell wall mechanics, but still apply the growth on edges of the mesh independently [4]. Such growth description will fail to be mesh-independent and will not be able to properly take into account anisotropies in material variables or in stresses. Most similar to our approach is the 2D element growth described in [6], where strain was applied as growth signal. The main difference is our reference system invariant description, more easily handling properties such as the growth threshold, and hence a more general methodology to add growth signals from strains, stresses, morphogens or other signals.

The need for detailed mechanical models is increasingly apparent for growth models in biology. Our work provides a recipe to include growth in such simulations, and its generality in terms of possibilities to include various input signals and feedbacks presents an important step towards models of plant morphogenesis over long time scales. This will, in example, allow for investigating large deformations in self-organizing patterning models combining hormonal and mechanical signals [24, 17]. The connection to the cells make it possible to connect the mechanical and growth description to 4D cellular data generated for example by confocal microscopy. As such, our description represents an essential model development within Computational Morphodynamics. 


\section{Author contributions}

Conceived and designed the experiments: BB HJ. Performed the experiments: BB. Analyzed the data: BB PK HJ. Wrote the paper: BB PK HJ.

\section{Acknowledgements}

This work was supported by the Swedish Research Council (VR2013:4632), the Gatsby Charitable Foundation (GAT3395/PR4), and the Knut and Alice Wallenberg Foundation via project ShapeSystems (KAW2012.0050).

\section{References}

[1] Karen Alim, Olivier Hamant, and Arezki Boudaoud. Regulatory role of cell division rules on tissue growth heterogeneity. Front Plant Sci, 3:174, Jan 2012.

[2] D Ambrosi, GA Ateshian, EM Arruda, SC Cowin, J Dumais, A Goriely, GA Holzapfel, JD Humphrey, R Kemkemer, E Kuhl, and JE Olberding. Perspectives on biological growth and remodeling. J Mech Phys Solids, 59:863-883, 2011.

[3] Tobias I Baskin. Anisotropic expansion of the plant cell wall. Annu Rev Cell Dev Biol, 21:203-22, Jan 2005.

[4] George W Bassel, Petra Stamm, Gabriella Mosca, Pierre Barbier de Reuille, Daniel J Gibbs, Robin Winter, Ales Janka, Michael J Holdsworth, and Richard S Smith. Mechanical constraints imposed by 3D cellular geometry and arrangement modulate growth patterns in the Arabidopsis embryo. Proc Natl Acad Sci USA, page 201404616, May 2014.

[5] S. Besson and J. Dumais. Universal rule for the symmetric division of plant cells. Proc Natl Acad Sci USA, 108(15):6294-9, Mar 2011.

[6] Frédéric Boudon, Jérôme Chopard, Olivier Ali, Benjamin Gilles, Olivier Hamant, Arezki Boudaoud, Jan Traas, and Christophe Godin. A computational framework for 3D mechanical modeling of plant morphogenesis with cellular resolution. PLoS Comp Biol, 11(1):e1003950, January 2015.

[7] Frédéric Boudon, Christophe Pradal, Thomas Cokelaer, Przemyslaw Prusinkiewicz, and Christophe Godin. L-py: an L-system simulation framework for modeling plant architecture development based on a dynamic language. Front Plant Sci, 3:76, 2012.

[8] Behruz Bozorg, Pawel Krupinski, and Henrik Jönsson. Stress and strain provide positional and directional cues in development. PLoS Comp Biol, 10:e1003410, 2014.

[9] Siobhan Braybrook and Henrik Jönsson. Shifting foundations: the mechanical cell wall and development. Curr Opin Plant Biol, 29:115-120, Jan 2016.

[10] Siobhan A Braybrook and Alexis Peaucelle. Mechano-chemical aspects of organ formation in Arabidopsis thaliana: the relationship between auxin and pectin. PloS ONE, 8(3):e57813, 2013.

[11] Daniel J Cosgrove. Growth of the plant cell wall. Nat Rev Mol Cell Biol, 6(11):850-61, Nov 2005.

[12] Daniel J Cosgrove. Plant cell wall extensibility: connecting plant cell growth with cell wall structure, mechanics, and the action of wall-modifying enzymes. J Exp Bot, 67(2):463-476, January 2016.

[13] R.J. Dyson, L.R. Band, and O.E. Jensen. A model of crosslink kinetics in the expanding plant cell wall: Yield stress and enzyme action. J Theor Biol, 307:125 - 136, 2012.

[14] John A Fozard, Michael Lucas, John R King, and Oliver E Jensen. Vertex-element models for anisotropic growth of elongated plant organs. Front PLant Sci, 4:233, 2013.

[15] S C Fry. Cellulases, hemicelluloses and auxin-stimulated growth: a possible relationship. Phys Plant, 75:532-536, 1989. 
[16] Olivier Hamant, Marcus G Heisler, Henrik Jönsson, Pawel Krupinski, Magalie Uyttewaal, Plamen Bokov, Francis Corson, Patrik Sahlin, Arezki Boudaoud, Elliot M Meyerowitz, Yves Couder, and Jan Traas. Developmental patterning by mechanical signals in arabidopsis. Science, 322(5908):1650-1655, Dec 2008.

[17] Marcus G Heisler, Olivier Hamant, Pawel Krupinski, Magalie Uyttewaal, Carolyn Ohno, Henrik Jönsson, Jan Traas, and Elliot M Meyerowitz. Alignment between pin1 polarity and microtubule orientation in the shoot apical meristem reveals a tight coupling between morphogenesis and auxin transport. PLoS Biol, 8(10):e1000516, Jan 2010.

[18] Nathan Hervieux, Mathilde Dumond, Aleksandra Sapala, Anne-Lise Routier-Kierzkowska, Daniel Kierzkowski, Adrienne H K Roeder, Richard S Smith, Arezki Boudaoud, and Olivier Hamant. A Mechanical Feedback Restricts Sepal Growth and Shape in Arabidopsis. Curr Biol, 26:1019$1028,2016$.

[19] Richard Kennaway, Enrico Coen, Amelia Green, and Andrew Bangham. Generation of diverse biological forms through combinatorial interactions between tissue polarity and growth. PLoS Comp Biol, 7(6):e1002071, June 2011.

[20] D. Kierzkowski, N. Nakayama, A.-L. Routier-Kierzkowska, A. Weber, E. Bayer, M. Schorderet, D. Reinhardt, C. Kuhlemeier, and R. S. Smith. Elastic Domains Regulate Growth and Organogenesis in the Plant Shoot Apical Meristem. Science, 335(6072):1096-1099, Mar 2012.

[21] Erika E Kuchen, Samantha Fox, Pierre Barbier De Reuille, Richard Kennaway, Sandra Bensmihen, Jerome Avondo, Grant M Calder, Paul Southam, Sarah Robinson, Andrew Bangham, and Enrico Coen. Generation of leaf shape through early patterns of growth and tissue polarity. Science, 335(6072):1092-1096, March 2012.

[22] James A Lockhart. An analysis of irreversible plant cell elongation. J Theor Biol, 8(2):264-275, March 1965.

[23] Prince Marowa, Anming Ding, and Yingzhen Kong. Expansins: roles in plant growth and potential applications in crop improvement. Plant Cell Rep, 35(5):949-965, May 2016.

[24] Alan C Newell, Patrick D Shipman, and Zhiying Sun. Phyllotaxis: cooperation and competition between mechanical and biochemical processes. J Theor Biol, 251(3):421-439, Apr 2008.

[25] Abdolmadjid Nili, Hojae Yi, Vincent H Crespi, and Virendra M Puri. Examination of biological hotspot hypothesis of primary cell wall using a computational cell wall network model. Cellulose, 22(2):1027-1038, 2015.

[26] Kazuhiko Nishitani and Yoshio Masuda. Auxin-Induced Changes in the Cell Wall Xyloglucans: Effects of Auxin on the Two Different Subtractions of Xyloglucans in the Epicotyl Cell Wall of Vigna angularis. Plant Cell Phys, 24(3):345-355, April 1983.

[27] Yong Bum Park and Daniel J Cosgrove. Xyloglucan and its interactions with other components of the growing cell wall. Plant Cell Phys, 56(2):180-194, February 2015.

[28] Alexis Peaucelle, Siobhan A Braybrook, Laurent Le Guillou, Emeric Bron, Cris Kuhlemeier, and Herman Höfte. Pectin-induced changes in cell wall mechanics underlie organ initiation in Arabidopsis. Curr Biol, 21(20):1720-1726, October 2011.

[29] Alexis Peaucelle, Raymond Wightman, and Herman Höfte. The Control of Growth Symmetry Breaking in the Arabidopsis Hypocotyl. Curr Biol, 25(13):1746-1752, June 2015.

[30] P. Prusinkiewicz and A. Lindenmeyer. Algorithmic Beauty of Plants. Springer, New York, 1990.

[31] D L Rayle and R E Cleland. The Acid Growth Theory of auxin-induced cell elongation is alive and well. Plant Phys, 99(4):1271-1274, August 1992.

[32] R L Robertson. Boundary identifiability of residual stress via the dirichlet to neumann map. Inverse Probl, 13:1107-1119, 1997.

[33] EK Rodriguez, A Hoger, and AD McCulloc. Stress-dependent finite growth in soft elastic tissue. J Biomech, 27:455-467, 1994.

[34] Patrik Sahlin and Henrik Jönsson. A modeling study on how cell division affects properties of epithelial tissues under isotropic growth. PLoS ONE, 5(7):e11750, Jan 2010.

[35] Arun Sampathkumar, Pawel Krupinski, Ray Wightman, P Malini, A Berquand, A Boudaoud, 
O Hamant, Henrik Jönsson, and Elliot M Meyerowitz. Subcellular and supracellular mechanical stress prescribes cytoskeleton behavior in arabidopsis cotyledon pavement cells. eLife, 3:e01967, 2014.

[36] Massimiliano Sassi, Olivier Ali, Frédéric Boudon, Gladys Cloarec, Ursula Abad, Coralie Cellier, Xu Chen, Benjamin Gilles, Pascale Milani, Jiří Friml, Teva Vernoux, Christophe Godin, Olivier Hamant, and Jan Traas. An Auxin-Mediated Shift toward Growth Isotropy Promotes Organ Formation at the Shoot Meristem in Arabidopsis. Curr Biol, 24(19):2335-2342, September 2014.

[37] Susanna Sauret-Güeto, Katharina Schiessl, Andrew Bangham, Robert Sablowski, and Enrico Coen. JAGGED controls Arabidopsis petal growth and shape by interacting with a divergent polarity field. PLoS Biol, 11(4):e1001550, 2013.

[38] Peter Schopfer, Anja Liszkay, Michael Bechtold, Gitta Frahry, and Andrea Wagner. Evidence that hydroxyl radicals mediate auxin-induced extension growth. Planta, 214(6):821-828, April 2002.

[39] Risto Sievänen, Christophe Godin, Theodore M DeJong, and Eero Nikinmaa. Functionalstructural plant models: a growing paradigm for plant studies. Ann Bot, 114(4):599-603, September 2014.

[40] R Skalak, G Dasgupta, M Moss, E Otten, P Dullemeijer, and H Vilmann. Analytical description of growth. J Theor Biol, 94:555-577, 1982.

[41] Chris Somerville. Cellulose Synthesis in Higher Plants. Annu. Rev Cell Dev Biol, 22:5378, October 2006.

[42] Larry A. Taber. Biomechanics of growth, remodeling, and morphogenesis. Appl Mech Rev, 48(8):487-545, Aug 1995.

[43] Alan M Turing. The chemical basis of morphogenesis. Philos Trans $R$ Soc Lond B, 237:37-72, 1952.

[44] Sebastian Wolf, Jozef Mravec, Steffen Greiner, Gregory Mouille, and Herman Höfte. Plant cell wall homeostasis is mediated by brassinosteroid feedback signaling. Curr Biol, 22:1732 - 1737, 2012 .

[45] T Zhang, Y Zheng, and D J Cosgrove. Spatial organization of cellulose microfibrils and matrix polysaccharides in primary plant cell walls as imaged by multichannel atomic force microscopy. Plant J, 85:179-192, 2016. 